\title{
A Lightweight Rao-Cauchy Detector for Additive Watermarking in the DWT-Domain
}

\author{
Roland Kwitt, Peter Meerwald, Andreas Uhl
}

\author{
Dept. of Computer Sciences, \\ University of Salzburg, \\ Austria
}

E-Mail: \{rkwitt, pmeerw, uhl\}@cosy.sbg.ac.at, Web: http://www.wavelab.at 


\section{Overview}

1. Introduction

2. Distribution of DWT subband coefficients

3. Cauchy distribution

4. Rao hypothesis test

5. Results 


\section{Introduction}

- Watermarking embeds a imperceptible yet detectable signal in multimedia content

- Blind watermarking detection does not have access to the unwatermarked host signal, thus host interferes with watermark detection

- Transform domains (DCT, DWT) facilitate perceptual and statistical modeling of the host

- Straightforward linear correlation detector only optimal for Gaussian host; DCT and DWT coefficient do not obey Gaussian law in general 


\section{Watermark Detection in Previous Work}

- Using Likelihood ratio test (LRT)

- host signal coefficients (DCT, DWT) modeled by GGD [Hernández et al., 2000]

- host signal coefficients (DCT) modeled by Cauchy distribution [Briassouli et al., 2005]

- LRT is optimal, but assumes that watermark power is known

- Using Rao test

- GGD host model [Nikolaidis and Pitas, 2003]

- Rao test makes no assumption on watermark power, but is only asymptotically equivalent to the GLRT

- GGD parameter estimation is computationally expensive 


\section{Distribution of DWT detail subband coefficients}

- GGD model known to fit DCT AC and DWT detail subband coefficients

- GGD parameters expensive to compute

- Often set GGD shape parameter to fixed value (eg. 0.5 or 0.8 for DCT/DWT coefficients)

- Alternative: Cauchy distribution 


\section{Cauchy Distribution}

- Cauchy has been applied to blind DCT-domain spread-spectrum watermarking [Briassouli et al., 2005]

- Cauchy distribution PDF

$$
p(x \mid \gamma, \delta)=\frac{1}{\pi} \frac{\gamma}{\gamma^{2}+(x-\delta)^{2}},
$$

with location parameter $-\infty<\delta<\infty$ and shape parameter $\gamma>0$

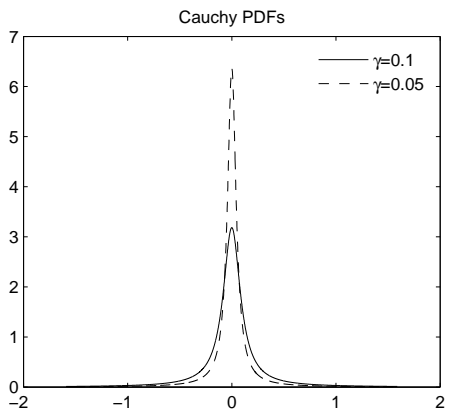




\section{Q-Q Plots of DWT Detail Subband Coefficients}

Decomposition level 2, horizontal orientation ( $H_{2}$ subband)
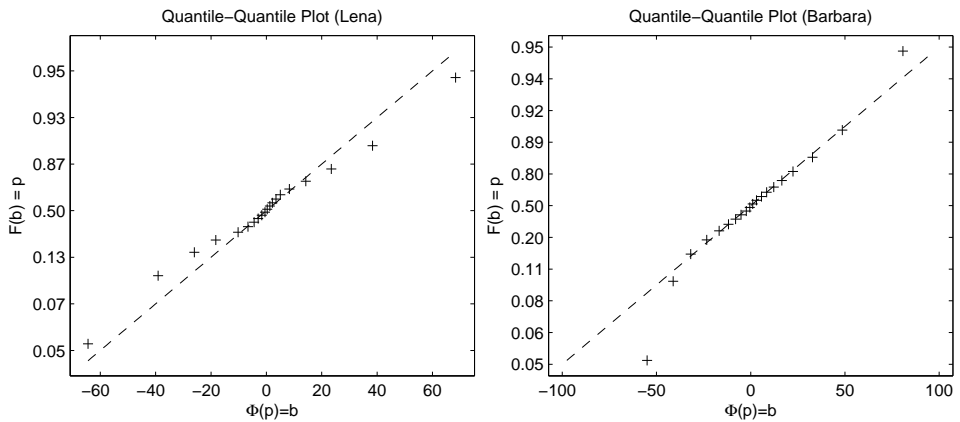


\section{Detection Problem}

- Consider DWT detail subband coefficients as i.i.d. random variables following a Cauchy distribution with parameters $\gamma$ and $\delta=0$

- Want to detect deterministic signal of unknown amplitude (the watermark scaled by strength parameter $\alpha$ ) in Cauchy distributed noise (the host signal)

$$
\begin{aligned}
& \mathcal{H}_{0}: \alpha=0, \gamma \text { (no/other watermark) } \\
& \mathcal{H}_{1}: \alpha \neq 0, \gamma \text { (watermarked) }
\end{aligned}
$$




\section{Rao Hypothesis Test}

- Two-sided composite hypothesis testing problem with one nuisance parameter $\gamma$

- In contrast to GLRT, Rao test does not require to estimate unknown parameter $\alpha$ under $\mathcal{H}_{1}$

- For symmetric PDFs [Kay, 1989], the Rao test statistic for our watermark detection problem can be written as

$$
\rho(\mathbf{y})=\left[\left.\sum_{i=1}^{N} \frac{\partial \log p(y[i]-\alpha w[i], \hat{\gamma})}{\partial \alpha}\right|_{\alpha=0}\right]^{2} \mathbf{I}_{\alpha \alpha}^{-1}(0, \hat{\gamma})
$$

$p(\cdot)$ denotes the Cauchy PDF, $\hat{\gamma}$ is the MLE of the Cauchy shape parameter, $\mathbf{I}_{\alpha \alpha}^{-1}$ is an element of the Fisher Information matrix 


\section{Detection Statistic}

After simplifications (inserting the Cauchy PDF and determining $\left.\mathrm{I}_{\alpha \alpha}^{-1}(0, \hat{\gamma})\right)$, the detection statistic becomes

$$
\rho(\mathbf{y})=\left[\sum_{t=1}^{N} \frac{y[t] w[t]}{\hat{\gamma}^{2}+y[t]^{2}}\right]^{2} \frac{8 \hat{\gamma}^{2}}{N}
$$

with the asymptotic property

$$
\rho \stackrel{a}{\sim} \begin{cases}\chi_{1}^{2}, & \text { under } \mathcal{H}_{0} \\ \chi_{1, \lambda}^{2}, & \text { under } \mathcal{H}_{1}\end{cases}
$$

$\chi_{1, \lambda}^{2}$ denotes the non-central $\chi^{2}$ distribution with non-centrality parameter $\lambda$ 


\section{Detection Responses under $\mathcal{H}_{0}$ and $\mathcal{H}_{1}$}

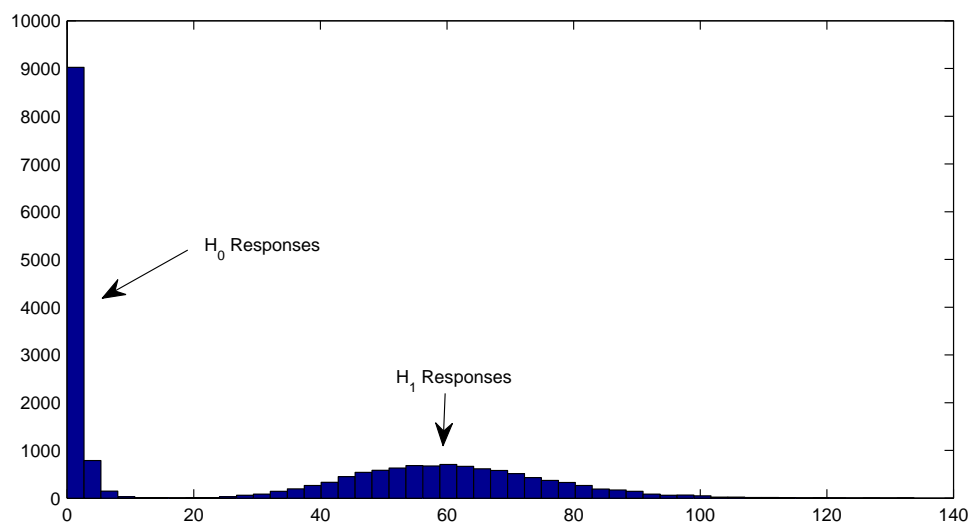




\section{Detection Probability}

- Since the distribution of the detector response $\rho$ under $\mathcal{H}_{0}$ and $\mathcal{H}_{1}$ is known, we can express the probability of false-alarm $\left(P_{f}\right)$, detection $\left(P_{d}\right)$ and miss $\left(P_{m}\right)$ as

$$
\begin{aligned}
& \qquad P_{f}=\mathbb{P}\left\{\rho>T \mid \mathcal{H}_{0}\right\}=\mathrm{Q}_{\chi_{1}^{2}}(T)=2 \mathrm{Q}(\sqrt{T}) \\
& P_{m}=1-P_{d}=1-\mathbb{P}\left(\rho>T \mid \mathcal{H}_{1}\right)=1-\mathrm{Q}(\sqrt{T}-\sqrt{\lambda})+\mathrm{Q}(\sqrt{T}+\sqrt{\lambda}) \\
& \text { where } T \text { denotes the detection threshold and } Q \text { is used to } \\
& \text { express right-tail probabilities of the Gaussian distribution. } \\
& \text { - The ROC can be plotted using }
\end{aligned}
$$

$$
P_{m}=1-\mathrm{Q}\left(\mathrm{Q}^{-1}\left(P_{f} / 2\right)-\sqrt{\lambda}\right)-\mathrm{Q}\left(\mathrm{Q}^{-1}\left(P_{f} / 2\right)+\sqrt{\lambda}\right)
$$

where we have expressed $P_{m}$ depending on $P_{f}$. 


\section{Host Signal Parameter Estimation}

To determine the MLEs for the Cauchy or GGD shape parameter, we have to solve

$$
\frac{1}{N} \sum_{t=1}^{N} \frac{2}{1+(x[t] / \hat{\gamma})^{2}}-1=0 \quad \text { (Cauchy) }
$$

or

$$
\begin{array}{r}
1+\frac{\psi(1 / \hat{c})+\log \left(\frac{\hat{c}}{N} \sum_{t=1}^{N}|x[t]|^{\hat{c}}\right)}{\hat{c}} \\
-\frac{\sum_{t=1}^{N}|x[t]|^{\hat{c}} \log (|x[t]|)}{\sum_{t=1}^{N}|x[t]|^{\hat{c}}}=0
\end{array}
$$

numerically. Approximately the same number of iterations are necessary (Newton-Raphson), however the computation effort is much higher for the GGD. 


\section{Detector Comparison: Computational Effort}

Number of arithmetic operations to compute detection statistic for signal of length $N$

\begin{tabular}{|l||c|c|c|c|}
\hline \multicolumn{1}{|l||}{ Detector } & \multicolumn{4}{c|}{ Operations } \\
&,+- & $\times, \div$ & pow, log & abs, sgn \\
\hline LC & $\mathrm{N}$ & $\mathrm{N}$ & & \\
\hline Rao-Cauchy & $2 \mathrm{~N}$ & $2 \mathrm{~N}+4$ & & \\
\hline Rao-GGD [Nikolaidis and Pitas, 2003] & $2 \mathrm{~N}$ & $3 \mathrm{~N}+1$ & $2 \mathrm{~N}$ & $3 \mathrm{~N}$ \\
\hline LRT-GGD [Hernández et al., 2000] & $3 \mathrm{~N}$ & 2 & $2 \mathrm{~N}+1$ & $2 \mathrm{~N}$ \\
\hline LRT-Cauchy [Briassouli et al., 2005] & $4 \mathrm{~N}$ & $5 \mathrm{~N}$ & $\mathrm{~N}$ & \\
\hline
\end{tabular}




\section{Rao-Cauchy Detector: Advantages / Disadvantages}

+ Easier parameter estimation for Cauchy distribution over GGD

+ Rao detection statistic requires less computational effort than LRT

+ No unknown parameters in the asymptotic PDF under $\mathcal{H}_{0}$ (constant false-alarm rate detector)

+ No knowledge of embedding strength required for computation of detection statistic

- Rao test only asymptotically equivalent to GLRT (no optimality associated with GLRT)

- Cauchy is a rough approximation of DWT detail subband statistics, especially in the tail regions (too heavy) 


\section{Detection Performance: Experimental Results}

\section{Embedding with $25 \mathrm{~dB}$ DWR}

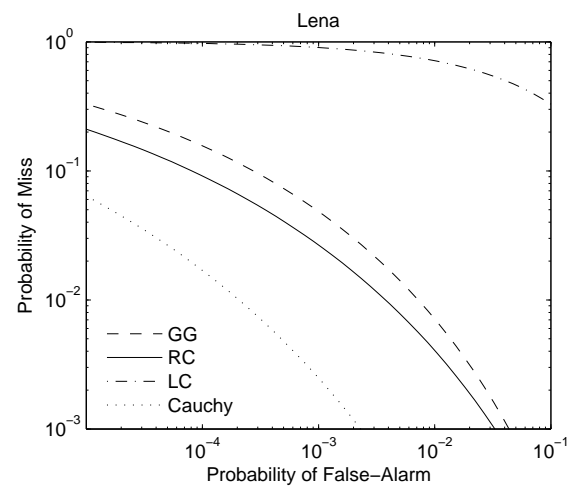

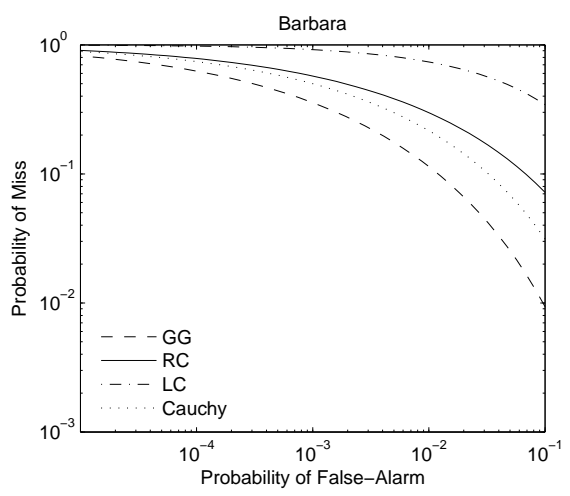




\section{JPEG Compression Attack}

\section{JPEG compression, $Q=50$; embedding DWR $20 \mathrm{~dB}$}
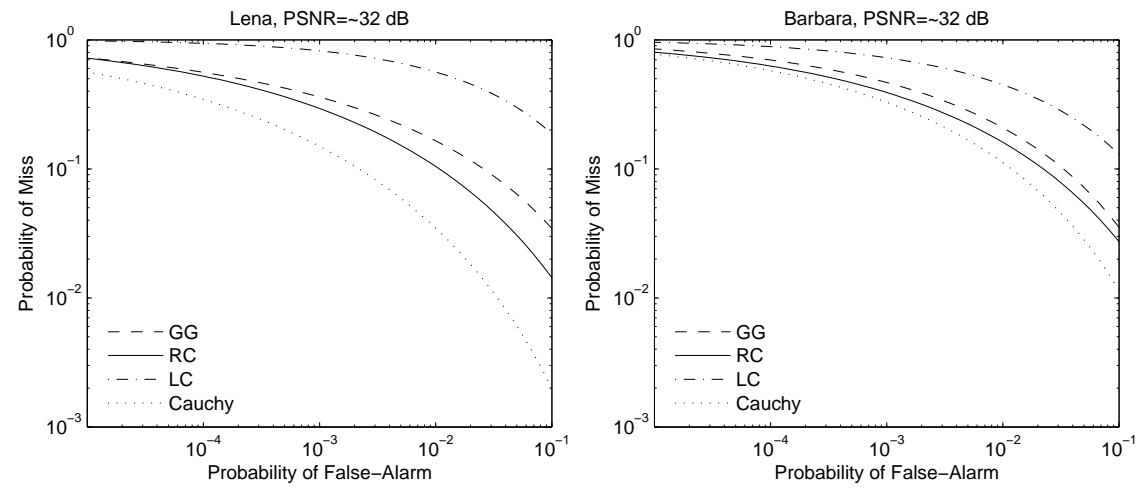


\section{JPEG2000 Compression Attack}

Jasper JPEG2000 codec, 2.4 bpp; embedding DWR 23 dB
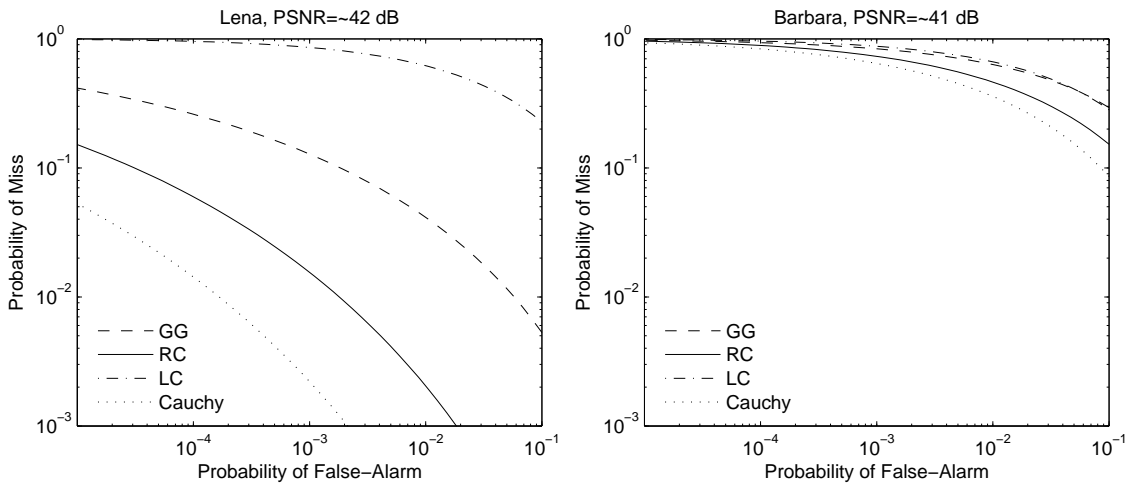


\section{Conclusion}

- DWT detail subband coefficients can be modeled by one-parameter Cauchy distribution

- Proposed Rao hypothesis test for Cauchy host data

- Parameter estimation of the Cauchy distribution is less expensive than for the GGD

- Computation of detection statistic for the Rao-Cauchy test more efficient than the LRT conditioned to the GGD or Cauchy distribution

- Rao-Cauchy detector has competitive detection performance

- Source code available on request: http://wavelab.at/sources 


\section{References}

园 Briassouli, A., Tsakalides, P., and Stouraitis, A. (2005).

Hidden messages in heavy-tails: DCT-domain watermark detection using alpha-stable models.

IEEE Transactions on Multimedia, 7(4):700-715.

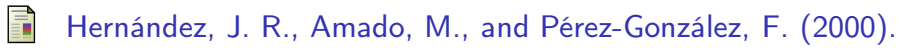

DCT-domain watermarking techniques for still images: Detector performance analysis and a new structure.

IEEE Transactions on Image Processing, 9(1):55-68.

Kay, S. M. (1989).

Asymptotically optimal detection in incompletely characterized non-gaussian noise.

IEEE Transactions on Acoustics, Speech and Signal Processing, 37(5):627-633.

Nikolaidis, A. and Pitas, I. (2003).

Asymptotically optimal detection for additive watermarking in the DCT and DWT domains.

IEEE Transactions on Image Processing, 12(5):563-571. 